\title{
Kinderschutz und Schutzkonzepte im klinischen und ambulanten Bereich
}

\author{
Jörg M. Fegert und Ulrike Hoffmann \\ Klinik für Kinder- und Jugendpsychiatrie/Psychotherapie, Universitätsklinikum Ulm
}

\begin{abstract}
Zusammenfassung: Die Auseinandersetzung mit Fällen von (sexualisierter) Gewalt gegen Kinder und Jugendliche sowie die Entwicklung von Schutzkonzepten war in den Institutionen des medizinisch-therapeutischen Bereiches über lange Zeit ein eher marginalisiertes Thema. Mit der Verankerung der Verpflichtung zur Erstellung von Schutzkonzepten gegen (sexualisierte) Gewalt in der Qualitätsmanagement-Richtlinie des Gemeinsamen Bundesausschusses (G-BA) müssen sich jedoch nun alle Kliniken und Praxen dieser Thematik stellen. Der vorliegende Themenschwerpunkt gibt einen Überblick über Daten und Zahlen (sexueller) Übergriffe gegen Kinder und Jugendliche in medizinischen Institutionen und beschäftigt sich mit den Ursachen von Übergriffen durch Angehörige der Heilberufe sowie den daraus folgenden notwendigen Interventionen. Weiteres Thema ist die Entwicklung von Schutzkonzepten gegen (sexuelle) Gewalt. Es werden Hinweise zum Aufbau sowie zur praktischen Umsetzung im klinischen und ambulanten Bereich gegeben.
\end{abstract}

Schlüsselwörter: Kinderschutz, Schutzkonzeptentwicklung, sexualisierte Gewalt, Gewalt, medizinische Institutionen

Child Protection and Safeguarding Measures in Inpatient and Outpatient Care

Abstract: Cases of (sexualized) abuse against children and adolescents and the development of safeguarding measures have long been marginalized by institutions in the medical-therapeutic sector. However, with the adoption of the quality management guidelines of the Federal Joint Committee (G-BA), all clinics and outpatient settings now have to face this issue and are obliged to develop safeguarding measures against (sexualized) violence. This issue gives an overview of the data and statistics regarding (sexual) assaults against children and adolescents in medical-therapeutic institutions and describes the causes of assaults by health care professionals as well as the resulting necessary interventions. Another topic is the development of safeguarding measures against (sexual) abuse and violence. Information is provided concerning the structure and practical implementation of safeguarding measures in both clinical and outpatient settings.

Keywords: child protection, development of safeguarding measures, child sexual abuse, violence, medical-therapeutic institutions

Die Auseinandersetzung mit Fällen von (sexualisierter) Gewalt gegen Kinder und Jugendliche sowie die Entwicklung von Schutzkonzepten war in den Institutionen des medizinisch-therapeutischen Bereiches über lange Zeit ein kaum wahrgenommenes Thema. Im Juni 2019 führten wir gemeinsam mit dem Unabhängigen Beauftragten für Fragen des sexuellen Kindesmissbrauchs, Herrn Rörig, und der Deutschen Krankenhausgesellschaft zu dieser Thematik die erste bundesweite Veranstaltung in Berlin durch. Die Veranstaltung fand einen Tag nach der Verurteilung des Krankenpflegers Niels Högel statt, der wegen der Tötung von 85 Patient_innen zu einer lebenslangen Freiheitsstrafe verurteilt wurde, was die Notwendigkeit der Auseinandersetzung mit der Thematik von Gewalt in medizinischen Institutionen zusätzlich unterstrich. Bei einer Pressekonferenz im Rahmen der Veranstaltung stand auch die Frage im Raum, wie es zu solchen Vorkommnissen in Kliniken kommen kann, wie können Bystander_innen eine Annahme darüber haben, dass etwas nicht stimmt und trotzdem werden die Vermutungen nicht weitergegeben und/oder das Handeln nicht unterbunden. Für alle Anwesenden überraschend und sehr überzeugend kündigte Herr Baum, der damalige Hauptgeschäftsführer der Deutschen Krankenhausgesellschaft in der Pressekonferenz dann an, dass er sich dafür einsetzen werde, dass sich der Gemeinsame Bundesausschuss im Rahmen der Qualitätsrichtlinie der Frage der Etablierung von Schutzkonzepten in medizinischen Einrichtungen annehmen werde. Dies ist im Nachgang erfolgt und seit dem November 2020 ist die Etablierung eines Schutzkonzeptes zur Prävention von und Hilfe bei Missbrauch und Gewalt mit der Verankerung in der QM-Richtlinie des G-BA 
für medizinische Einrichtungen nun verpflichtend. Formuliert wurde bezüglich des Zieles der entsprechenden Regelung: „Ziel ist es, Missbrauch und Gewalt insbesondere gegenüber vulnerablen Patientengruppen, wie beispielsweise Kindern und Jugendlichen oder hilfsbedürftigen Personen, vorzubeugen, zu erkennen, adäquat darauf zu reagieren und auch innerhalb der Einrichtung zu verhindern." (G-BA, 2020, S. 1) Für die Umsetzung wurden konkrete Elemente vorgegeben, wie z.B. die Durchführung einer Gefährdungsanalyse, ein Verhaltenskodex und ein Interventionsplan (G-BA, 2020).

Mit dieser Vorgabe müssen sich nun alle Praxen und Krankenhäuser, insbesondere wenn diese Kinder und Jugendliche behandeln, mit der Etablierung von Präventions- und Schutzmaßnahmen vor sexuellem Missbrauch und Gewalt beschäftigen.

Im Gegensatz zu den fortgesetzten Debatten um Aufarbeitung von Fällen sexualisierter Gewalt zum Beispiel im kirchlichen Bereich oder im Sport fehlt im Gesundheitsbereich bislang eine systematische Tradition des Fehler-Lernens unter Einbezug von Betroffenen. Generell ist deshalb sowohl im öffentlichen Bewusstsein, als auch bei den Fachkräften, die im Gesundheitswesen tätig sind, noch nicht generell von einem Vorverständnis dafür auszugehen, dass Krankenhäuser und Praxen Orte sind, die vulnerablen Patient_innen Schutz, Hilfe und Therapie bieten sollen, dass gleichzeitig aber diese Abhängigkeitsverhältnisse und die besondere Vulnerabilität auch von Täter_innen für Übergriffe genutzt werden können. Auch dass dies, ebenso wie auch in anderen institutionellen Bereichen nicht selten und häufig auch systematisch passiert, wird nicht zuletzt aufgrund der mangelnden Aufarbeitung zu wenig wahrgenommen.

Im Saarland wird nun erstmals ein staatlich finanziertes Aufarbeitungsprojekt im medizinischen Bereich durchgeführt werden. Dieses wird sicher über die Landesgrenzen hinaus Bedeutung erlangen, weil nicht, wie im entsprechenden politischen Untersuchungsausschuss, Fragen von individueller Schuld und persönlichem sowie institutionellem Versagen im Mittelpunkt stehen werden, sondern Fragen des Lernens aus den Geschehnissen und des Verstehens, wie ein solcher Verlauf möglich war, obwohl es frühzeitig Hinweise gegeben hat. Gerade solche Aufarbeitungen sind eine Voraussetzung dafür, wirksame Schutzmaßnahmen zu etablieren oder bereits bestehende zu optimieren.

Manche Träger, insbesondere die kirchlichen, waren, weil sie als Gesamtinstitution ja lange Zeit „am Pranger" standen, Vorreiter im Bereich der Prävention. Regelungen und Schutzkonzepte, z.B. im Bereich der Katholischen Kirche, mit der revidierten Präventionsordnung von 2020 haben explizit auch den Bereich katholischer Krankenhäuser und die Vulnerabilität anderer
Gruppen, wie die von Erwachsenen mit Behinderung etc., im Blick. Gleichwohl gilt auch hier, dass gut gemeinte, top-down etablierte, in Handbüchern dokumentierte und den jeweiligen Vorgaben entsprechende Schutzkonzepte noch kein Verständnis vor Ort, kein Bewusstsein für die institutionsspezifischen Risiken oder keine Haltung zum Schutz vor Gewalt garantieren. Versucht man dann bottom-up in der Praxis an der Thematik zu arbeiten, zeigen sich häufig Verwerfungen in den Teams, alte offene Rechnungen, teilweise wird dann auch vor Ort deutlich, was unter den „Teppich gekehrt" worden ist und wer welches Wissen für sich behalten hat.

Die Beschäftigung mit (sexuellen) Übergriffen in Institutionen sollte sich darüber hinaus nicht nur mit Patient_innen als Betroffene beschäftigen, sondern auch die Mitarbeitenden fokussieren, da sexuelle Belästigungen durch Patient_innen oder Kolleg_innen ebenso ein Problem darstellen.

Die Thematik der Umsetzung von Schutzkonzepten birgt also Sprengstoff. Gleichzeitig sind viele Personen in Leitungsverantwortung, viele Praxisinhaber_innen in einer Zeit ausgebildet worden, wo diese Thematik in der Facharzt- oder der Psychotherapieausbildung noch eine völlig untergeordnete Rolle spielte. Notwendig ist deshalb, Informationen zum Ausmaß von (sexuellen) Übergriffen zu Risikofaktoren sowie zum Aufbau von Schutzkonzepten zu geben.

Neben der Perspektive „Institution als Schutzort“, also eine Institution, in der institutionelle Strukturen und Abläufe so gestaltet sind, dass Grenzüberschreitungen und Gewalt erkannt, benannt und Maßnahmen ergriffen werden, diese zu stoppen bzw. präventiv zu verhindern, muss auch die Perspektive der "Institution als Kompetenzort“ mitbedacht werden. Dies bedeutet, dass Kinder und Jugendliche, die von Gewalt betroffen sind, in medizinischen Einrichtungen kompetente Hilfe und Unterstützung finden müssen, ihre Schutzbedürfnisse im klinischen Alltag sichtbar gemacht werden sollten und ein Ort geschaffen wird, am dem sie es schaffen können sich anzuvertrauen (Rörig, 2019).

Unser Anliegen mit diesem Themenschwerpunkt ist es, durch Daten zu (sexuellen) Übergriffen gegen Patient_innen deutlich zu machen, dass es sich nicht um singuläre Skandale oder Ereignisse handelt, sondern dass diese nur die Spitze des Eisbergs darstellen und andererseits durch Schilderungen von Fallkonstellationen und daraus abgeleiteten Überlegungen zur Umsetzung von Schutzkonzepten einfühlbarer zu machen, wie nahe solche Grenzbereiche in der alltäglichen Praxis sind. Nur wenn es uns gelingt ein Bewusstsein zu schaffen, dass die zurecht privilegierten und geschützten Bereiche der Heilbehandlung auch spezifische, gefahrgeneigte Situationen sind, können 
wir nachhaltige Schutzprozesse initiieren, die in Krankenhäusern, Einrichtungen und Praxen gelebt und als Haltung tradiert werden.

\section{Literatur}

G-BA (Gemeinsamer Bundesausschuss) (2020). Beschluss des Gemeinsamen Bundesausschusses über eine Änderung der Qualitätsmanagement-Richtlinie (QM-RL): Vorgaben für die regelmäßige Erhebung und Darlegung des aktuellen Stands der Umsetzung und Weiterentwicklung von einrichtungsinternem Qualitätsmanagement sowie weitere Änderung in $\S 4$ der Richtlinie. Verfügbar unter https://www.g-ba.de/downloads/
39-261-4379/2020-07-16_QM-RL_Vorgaben-aktueller-Stand. pdf

Rörig, J.-W. (2019). Grußwort. In: Kappler, S., Hornfeck, F., Pooch, M.T., Kindler, H., Tremel, I. (2019). Kinder und Jugendliche besser schützen - der Anfang ist gemacht. Schutzkonzepte gegen sexuelle Gewalt in den Bereichen: Bildung und Erziehung, Gesundheit, Freizeit (S. 2 - 3). Verfügbar unter https://www.dji.de/ fileadmin/user_upload/bibs2019/28116_UBSKM_DJI_Ab schlussbericht.pdf

\section{Prof. Dr. Jörg M. Fegert}

Klinik für Kinder- und Jugendpsychiatrie/Psychotherapie Universitätsklinikum Ulm Steinhövelstraße 5 89073 Ulm

joerg.fegert@uniklinik-ulm.de 